\title{
Effectiveness of Grid Orthogonality on Body Fitted Curvilinear Coordinate System in CFD
}

\author{
Ichiro Nakane
}

\begin{abstract}
Body fitted curvilinear coordinate systems (usually abbreviated to "BFC") are widely used for calculating the flow around arbitrary complex bodies, but generally they are not orthogonal. The accuracy deteriorates if the departure from orthogonality is too large, and non-orthogonal coordinate systems make the application of boundary conditions more complex. In this study, the method for generating the orthogonal BFC based on the non-orthogonal BFC is suggested, and five kinds of calculation grids (one non-orthogonal and four orthogonal grids) are generated. The two-dimensional calculations were performed for the flow around a circular cylinder with these orthogonal and non-orthogonal grids. The calculated results were compared with the measurements and the analytic prediction. The validity of grid orthogonality and the method for generating the orthogonal BFC are confirmed.
\end{abstract}

Index Terms-Orthogonal curvilinear coordinate system, BFC, CFD, cylinder wake.

\section{INTRODUCTION}

Body-fitted curvilinear coordinate systems (usually abbreviated to "BFC") and the cut cell method are widely employed in the numerical calculation for the flow around arbitrary complex bodies. Of these, BFC have been used when the accurate result is needed. Now, in BFC, each coordinate system grid is abbreviated to "BFC grid" in following (refer to Fig. $1 \sim$ Fig. 3).

Thompson who is an authority on grid generation techniques proposed the versatile method of generation of BFC [1]. Furthermore, the BFC grid by his method has the particular advantage that the coordinate lines can be generated to closely approximate the streamlines and to be smooth, because this BFC grid is generated by solving elliptic partial differential equations like potential flow analysis. However, the coordinate lines of this BFC grid are usually non-orthogonal. Then, Thompson et al. wrote "Strict orthogonality is not necessary by involving proper normal derivatives", but after this sentence, they added "The accuracy deteriorates if the departure from orthogonality is too large". Accordingly, there is a possibility that the calculation accuracy with non-orthogonal body-fitted curvilinear coordinate systems (abbreviated to "NBFC") is low, with comparing to orthogonal body-fitted curvilinear coordinate systems (abbreviated to "OBFC"). In addition, NBFC grid makes the application of boundary conditions more complex.

For this reason, grid generation techniques have also been

Manuscript received December 5, 2012; revised February 6, 2013.

I. Nakane is with the Kanagawa Institute of Technology, Kanagawa, Japan (e-mail: inakane@me.kanagawa-it.ac.jp). put forward [3], which generate a three dimensional grid and guarantee the orthogonality of the coordinate lines. However, we can not found any papers that report the accuracy improvement effect by adding the orthogonality to NBFC.

Therefore, in this paper, the very simple integral method for generation of OBFC is proposed by modifying NBFC generated by the Thompson's method [1]. Using this OBFC, numerical calculations are carried out, and the calculation accuracy improved by the orthogonality of the coordinate lines is investigated. The calculation target is the flow around a circular cylinder arranged inside a rectangular pool. The four kinds of OBFC grids around a circular cylinder are generated from a NBFC grid by this integral method. In these four OBFC grids, the orthogonal regions from the cylindrical wall are different as follows: first 5-lines $\left(\xi_{o}=5\right), 15$-lines $\left(\xi_{O}=15\right), 25$-lines $\left(\xi_{O}=25\right)$ and 35 -lines $\left(\xi_{O}=35\right)$.

In this paper, the total five BFC grids (one NBFC and four OBFC grids) are generated and compared each other.

Therefore, we don't change the location of the boundary points and the coordinate lines along the flow direction, and we only change the number of orthogonal lines among these BFC grids to confirm the effectiveness of grid orthogonality.

\section{GENERATION OF OBFC GRIDS FROM A NBFC GRID}

In this study, a NBFC grid is generated according to Thompson et al. [1], and OBFC grids are generated from this NBFC grid. The flow field for calculation is assumed to be symmetric, and the BFC grids are generated for just only half of the flow field to shorten the computation time. The procedure for the OBFC grid generation is given below.

Following the Thompson's method [1], the distribution of $\xi$ and $\eta$ inside the boundary points is found by solving the Poisson equation given by (1) below, and the NBFC grid is generated as their contour lines. Now, $P$ and $Q$ in (1) are the control functions for attracting the coordinate lines to the designated lines and points. To help in understanding of the BFC, a schema of NBFC grid is also shown in Fig. 1.

$$
\left\{\begin{array}{l}
\xi_{x x}+\xi_{\mathrm{yy}}=P(\xi, \eta) \\
\eta_{x x}+\eta_{\mathrm{yy}}=Q(\xi, \eta)
\end{array}\right.
$$

As is clear from the above (1) and the below schema, the $\xi$ line $(\eta=$ constant line $)$ in the NBFC is distribution which closely approximates the streamline of the potential flow, because (1) becomes the Laplace equation in setting $P=0$. Therefore, for the improvement of the calculation accuracy, the $\xi$ line of the NBFC grid is left as it is, and the new $\zeta$ line orthogonal to the $\xi$ line is generated as follows. 


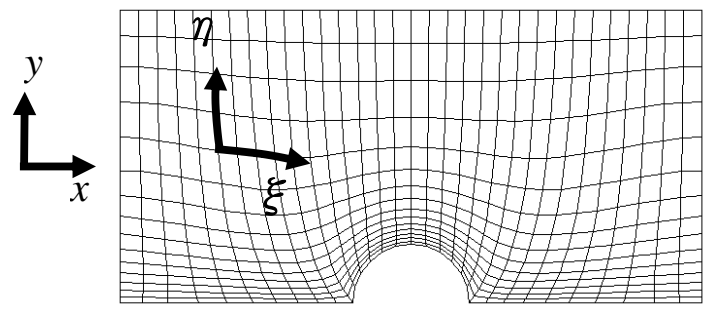

Fig. 1. Schema of non-orthogonal body fitted curvilinear coordinate system (NBFC grid)

The unit vector in the tangential direction to the $\xi$ line is given by the following equation.

$$
t^{(\xi)}=\frac{x_{\xi}}{\sqrt{x_{\xi}^{2}+y_{\xi}^{2}}} \boldsymbol{i}+\frac{y_{\xi}}{\sqrt{x_{\xi}^{2}+y_{\xi}^{2}}} \boldsymbol{j}
$$

Here, new variable $\zeta$ is defined in the following (3) as the potential quantity representing the unit vector in (2). The $\zeta$ contour lines are orthogonal with the $\xi$ lines, because the gradient lines and contour lines are orthogonal.

$$
d \zeta=\frac{x_{\xi}}{\sqrt{x_{\xi}^{2}+y_{\xi}^{2}}} d x+\frac{y_{\xi}}{\sqrt{x_{\xi}^{2}+y_{\xi}^{2}}} d y
$$

Furthermore, this (3) is transformed into (4) below, because of $d x=x_{\xi} d \xi+x_{\eta} d \eta \quad d y=y_{\xi} d \xi+y_{\eta} d \eta$.

$$
d \zeta=\frac{1}{\sqrt{x_{\xi}^{2}+y_{\xi}^{2}}}\left\{\left(x_{\xi}^{2}+y_{\xi}^{2}\right) d \xi+\left(x_{\xi} x_{\eta}+y_{\xi} y_{\eta}\right) d \eta\right\}
$$

Accordingly, by integrating (4), the $\zeta$ line orthogonal to the $\xi$ line can be obtained. However, in the actual calculation, this integration is performed on the $\xi$ line ( $\eta=$ constant line) according to the following (5), and the $\zeta$ value is obtained. The $\zeta$ line is generated as this contour line. Thus, the orthogonal technique in this study is fairly straightforward, when compared to other two dimensional methods, for example, the method proposed by Akcelik et al. [4] to generate an approximately orthogonal coordinate system.

$$
\begin{aligned}
\zeta & =\int \frac{1}{\sqrt{x_{\xi}^{2}+y_{\xi}^{2}}}\left\{\left(x_{\xi}^{2}+y_{\xi}^{2}\right) d \xi+\left(x_{\xi} x_{\eta}+y_{\xi} y_{\eta}\right) d \eta\right\} \\
& =\int \frac{1}{\sqrt{x_{\xi}^{2}+y_{\xi}^{2}}}\left(x_{\xi}^{2}+y_{\xi}^{2}\right) d \xi=\int \sqrt{x_{\xi}^{2}+y_{\xi}^{2}} d \xi
\end{aligned}
$$

Here, since the integration of (5) is the essence of the method proposed in this paper for generating an orthogonal coordinate system, the process involved in this integration, that is the details of the calculation process for the $\zeta$ value, are described below. First, at each grid point of the original NBFC grid, the values of $x_{\xi}$ and $y_{\xi}$ are calculated with the difference formula. Next, based on the calculated $x_{\xi}$ and $y_{\xi}$, numerical quadrature of (5) is performed on the $\xi$ line to find $\zeta$. Now, this numerical quadrature proceeds by the adding of values at each of the grid points, but numerical errors can easily become large with this kind of sequential adding. Accordingly, as is clear from Fig. 3 which is shown later, since the calculation grid is symmetrical, the integration advances from the center $\eta$ line to the left and right side to make the error small for the important area around the semicircle. This center $\eta$ line extends out from the midpoint of the semicircle to form the line of symmetry and this line is orthogonal to the $\xi$ line even for the NBFC grid.

Also, as is clear in the process to derive (5), in this OBFC grid, the $\xi$ lines and the $\zeta$ lines are conceptually same as the streamlines and the equipotential lines respectively. Therefore, as well as the original $\xi$ lines, but also the new $\zeta$ lines do not cross each other, and their variation is gradual. However, the coordinate lines orthogonal to the cylinder expand out radially, so the grid becomes extremely large with increasing distance away from the cylinder. In this paper, the $\zeta$ lines up to a certain fixed number from the cylinder wall are orthogonalized to the $\xi$ lines, and the remaining $\zeta$ lines become gradually asymptotic to the original $\eta$ lines. After all, we made the following 4 kinds of partial orthogonal grids to compare the calculation accuracy.

1) OBFC grid which is orthogonal up to 5 lines from the cylinder wall (written as $\xi_{O}=5$, the narrowest orthogonal region)

2) OBFC grid which is orthogonal up to 15 lines from the cylinder wall (written as $\xi o=15$ )

3) OBFC grid which is orthogonal up to 25 lines from the cylinder wall (written as $\xi_{O}=25$ )

4) OBFC grid which is orthogonal up to 35 lines from the cylinder wall (written as $\xi o=35$, the widest orthogonal region)

Here, the total number of $\xi$ lines is 66 , and the proportion of orthogonal lines for each OBFC grid become 7.5\% 53\% with respect to the total number of $\xi$ lines. The coordinate lines in the non-orthogonal region of the partial orthogonal grids (a) (d) are obtained using the moving average for the new orthogonal and the original non-orthogonal lines. Consequently, these coordinate lines in the non-orthogonal region of $\mathrm{BFC}$ grids are ultimately asymptotic to the coordinate lines of the NBFC. In other words, along with increasing distance from the cylinder, the OBFC grid matches up with the NBFC grid.

The OBFC grids generated using this method is shown in Fig. 2 and Fig. 3. In particular, the comparison of the original NBFC grid and the OBFC $\left(\xi_{o}=35\right)$ grid near the cylinder can easily be made in Fig. 2 which shows each quarter circle cut out and connected. The orthogonality of the OBFC grid in the vicinity of the cylinder is easy to see in this Fig. 2. In addition, from Fig. 2 and the detailed view in Fig. 3, it can also be verified that the coordinate lines for the orthogonal region and the non-orthogonal region join up smoothly. Now, as we can infer from Fig. 2, in an orthogonal coordinate system, it will be possible to make the grid shape change more slowly by appropriately moving the boundary points. Here, however, since the objective of this study is simply to investigate the effectiveness of grid orthogonality, the positions of the boundary points with the original $\mathrm{NBFC}$ and the OBFC have been made the same. 


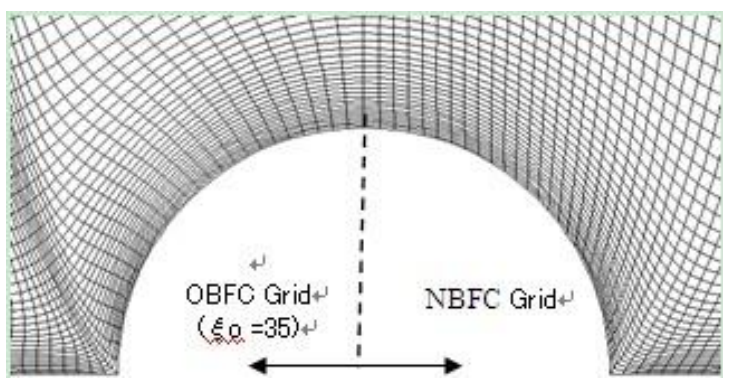

Fig. 2. Comparison between orthogonal (OBFC Grid $\xi_{o}=35$, left half side) and non-orthogonal (NBFC grid, right half side body fitted) curvilinear coordinate system

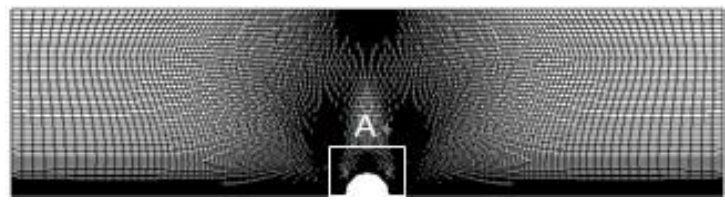

(a) Whole view

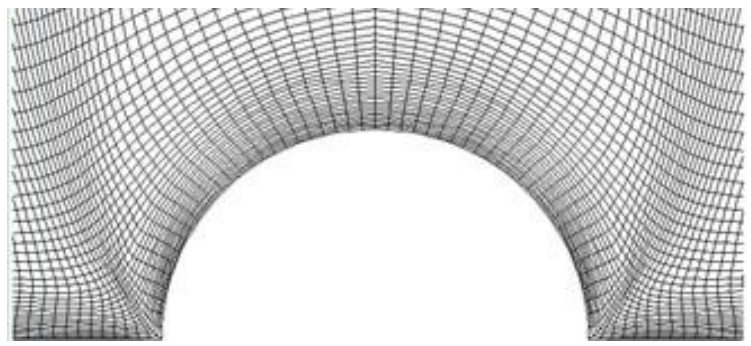

(b) Detail view of a-part

Fig. 3. Orthogonal body fitted curvilinear coordinate system (OBFC grid $\left.\xi_{o}=15\right)$

\section{NUMERICAL CALCULATION METHOD}

In this paper, using 4 different kinds of the new OBFC grids and the original NBFC grid, the effectiveness of the OBFC grid has been verified with the numerical calculation for the flow around a circular cylinder. An outline of the calculation method is listed below.

1) Governing equation : Stream function $\psi$ - Vorticity $\omega$ method

2) Discretization method: Finite volume method

3) Difference scheme : Convection term is an implicit method using the hybrid method, and diffusion term is the central difference method

4) Solution of the algebraic equation : Line-sequential method using the tridiagonal matrix method

5) Convergence criteria for iteration: The maximum absolute relative errors for each variables $\left|\left(\psi_{i, j}^{n+1}-\psi_{i, j}^{n}\right) / \psi_{i, j}^{n}\right|$ and $\left|\left(\omega_{i, j}^{n+1}-\omega_{i, j}^{n}\right) / \omega_{i, j}^{n}\right|$ is less than $10^{-4}$ ( $n$ : Iteration number, $i, j$ : Grid array index).

Now, the boundary conditions are as follows.

1) Cylinder wall : Non-slip wall

2) Inlet : Uniform velocity

3) Outlet : Fully developed flow (The gradient for each variable at the outlet is zero in the flow direction as following $\partial \psi /\left.\partial x\right|_{x_{\max }}=0$ and $\partial \omega /\left.\partial x\right|_{x_{\max }}=0$ )

4) Upper boundary : Slip wall

5) Lower boundary excluding the cylinder wall : Plane of symmetry (Symmetrical boundary)
Also, an overall grasp of the grid and the flow field is possible from Fig. 2 and Fig. 3, but quantitatively, a summary of it can be appended as follows.

1) Size of the calculation region : $16 d$ in the flow direction and $4 d$ in the width direction [ $d$ : Cylinder diameter]

2) Number of coordinate lines (grid points) : 224 in the flow direction and 65 in the width direction

3) Average distance of the first grid point away from the cylinder : $4 \times 10^{-2} d$ in the circumferential direction and $1 \times 10^{-2} d$ in the radial direction

Now, the scheme about the boundary condition and the discretization in this calculation conforms to the way of Thompson et al. [5]. As already described above, the calculation accuracy can be kept by this scheme except for extremely large non-orthogonal coordinate lines, and any techniques which can be used only for the orthogonal grid is not included in this scheme.

\section{RESULTS AND CONSIDERATIONS}

The calculated closed cylinder wake for the non-dimensional time $t^{*}=2.5\left(t^{*}=U t / d, U\right.$ : representative velocity, $t$ : real time) is shown in Fig. 4 to compare the visualization result measured by Bouard et al. [6]. In Fig. 4, figure (a) and (b) are results for $R e=550$, and figure (c) and (d) are results for $R e=3000$. Then, in these figures, the visualization results [6] are arranged in the upper half with the numerical calculation results in the lower half, and joined together into a single picture. The calculation results in Fig. 4 were obtained using the above-mentioned $\xi_{0}=35$ OBFC grid with the widest orthogonal region and also its original NBFC grid. Thus, the streamlines in the lower half for (a) and (c) are the calculation results using the $\xi_{o}=35 \mathrm{OBFC}$ grid, and the streamlines in the lower half for (b) and (d) are the calculation results using the NBFC grid. As is clear from this Fig. 4 for the position of main eddy core and the separation point, either calculated result is seen to agree well with the visualization results. However, when observed in detail, the differences in the length of the recirculation zone in the flow direction ( $L$ in the next Fig. 5) between the $\xi_{O}=35$ OBFC grid and the NBFC grid can be seen. This differences can be seen similarly for both $R e=550$ and $R e=3000$. In addition, for the calculation results using the NBFC grid, not just those for the $\xi_{o}=35$ OBFC grid, when also compared with the visualization results, the recirculation zone is long in the flow direction. In other words, we infer that the low calculation accuracy of the non-orthogonal coordinate system is also apparent qualitatively.

Thus, with the 4 different kinds of OBFC grid generated in this study and the original NBFC grid, the extent to which differences in the calculation results arise is investigated. The calculation results with each grid are judged through comparison of the measured geometrical parameters of the closed wake as shown in Fig. 5.

These results are shown in Fig. 6. Now, for the geometrical parameters in Fig. 5, we have, $a$ : the distance in the $x$ direction to the main eddy core from the rear side of the cylinder, $b$ : the distance between the main eddy core of the twin vortex, and $L$ : the length of the closed wake behind the cylinder along the center line. As is clear from Fig. 6, overall, 
the numerical calculation results and the visualization results of Bouard et al. [6] agree very well except for only $t^{*}=0.5$ of $b / 2 d$. This only difference is inferred as the combination of some factors, but the primary reason is conjectured that the closed wake has extremely elongated breadth as shown in Fig. 6(c). Consequently, the exact measurement and calculation of the breadth center of the eddy core is too difficult. For example, if assuming that the point $\mathrm{A}$ is the calculated eddy core, the calculation result agrees well with the measurement result as shown in Fig. 6(c). Further, taking in the effects due to differences in the grids, for the position of main eddy core, $a / d$ and $b / 2 d$, those differences are only slight. In addition, even with differences for the grids in $a / d$ and $b / 2 d$, we can conclude that these are random differences which show no constant trend according to the grid, but rather are considered to be associated with, for example, the reading accuracy of the main eddy core, or the convergence of the numerical calculation. However, regarding the problematic wake length $L / d$ in Fig. 4, there is a clear correlation in the calculated results through the area of the orthogonal region for the grid used in the calculations as shown in Fig. 6(a), and the differences with the visualization results are very conspicuous as the orthogonal region gets narrower. In particular, for $t^{*}=3.0$, whereas the calculation results with the $\xi_{O}=35$ OBFC grid agree with the visualization results and differ by less than $2 \%$, the results calculated for the NBFC grid differ from the visualization results by about $8 \%$. Moreover, these differences show a tendency to become more significant as $t^{*}$ gets larger. Now, the visible effects of the non-orthogonality of the grid appear for just $L / d$ and hardly appear at all for $a / d$ and $b / 2 d$, because the flow velocity is very slow near the eddy core. This is for the following reason. For the orthogonal coordinate system, the pseudodiffusion in the convection term is prevented, and a highly accurate solution is obtained. However, when the flow velocity is slow, because the diffusion is dominant, the advantage of the prevention of the pseudodiffusion can therefore be considered as small. Then, also for this reason, if $R e$ is small, we can predict that the advantage of the orthogonal grid is not easily reflected in the calculation results, even with the closed wake length $L / d$.

In fact, concerning this point, as confirmed by Fig. 7, which gives the results for the smaller $\operatorname{Re}=31$, the calculation results are roughly the same regardless of the grid, and agree well with the visualization results. Now for this reason, conversely, if $R e$ is large, we can infer that there will be a marked drop in calculation accuracy with the NBFC grid.

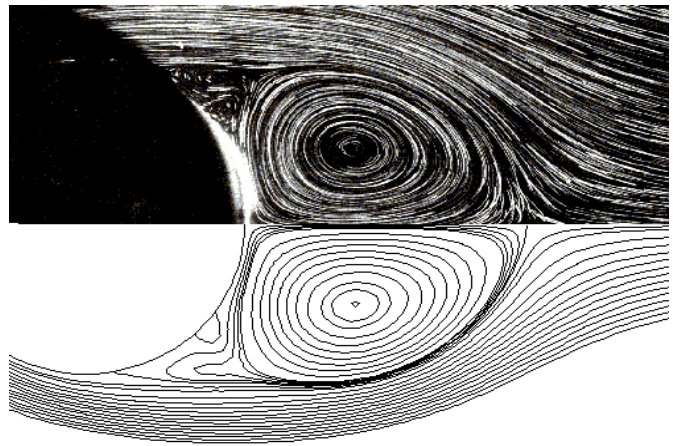

(a) $R e=550, t^{*}=2.5$, OBFC grid $(\xi o=35)$

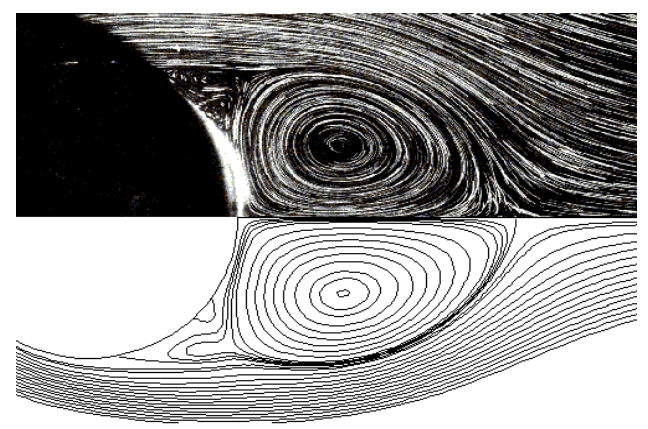

(b) $R e=550, t^{*}=2.5, \mathrm{NBFC}$ grid

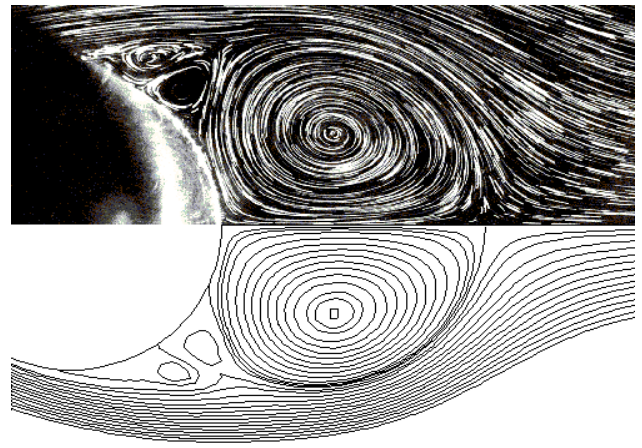

(c) $R e=3000, t^{*}=2.5, \mathrm{OBFC}$ grid $\left(\xi_{o}=35\right)$

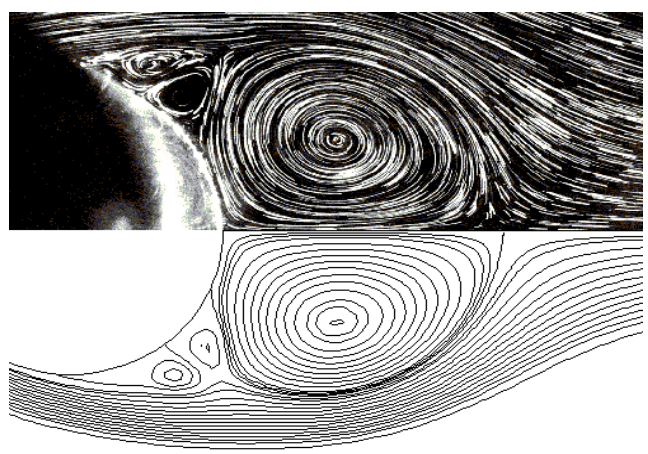

(d) $R e=3000, t^{*}=2.5, \mathrm{NBFC}$ grid

Fig. 4. Comparison between calculated and measured streamlines behind a circular cylinder. Upper half side: Flow visualization ${ }^{(6)}$, lower half side: Numerical calculation

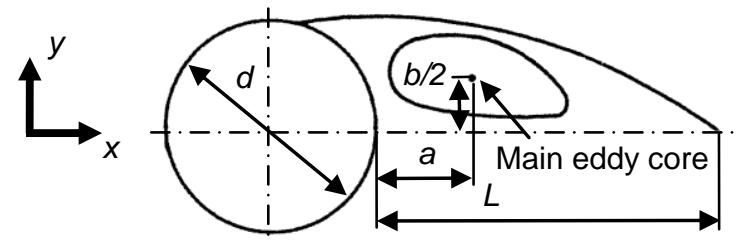

Fig. 5. Geometrical parameters of the closed cylinder wake

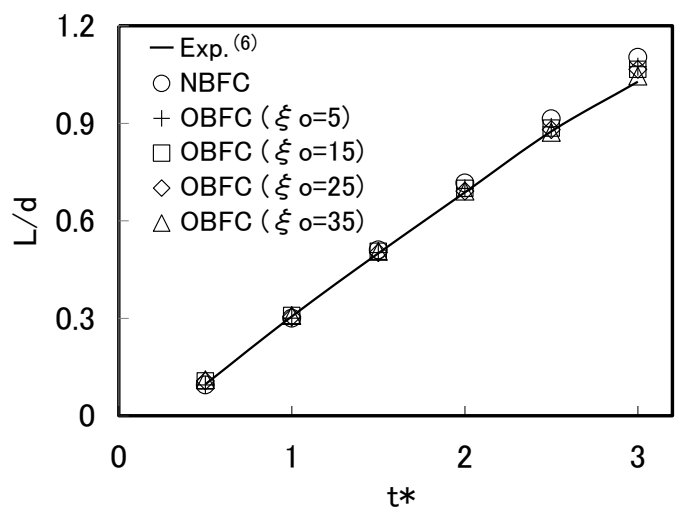

(a) Closed wake length $L / d$ 


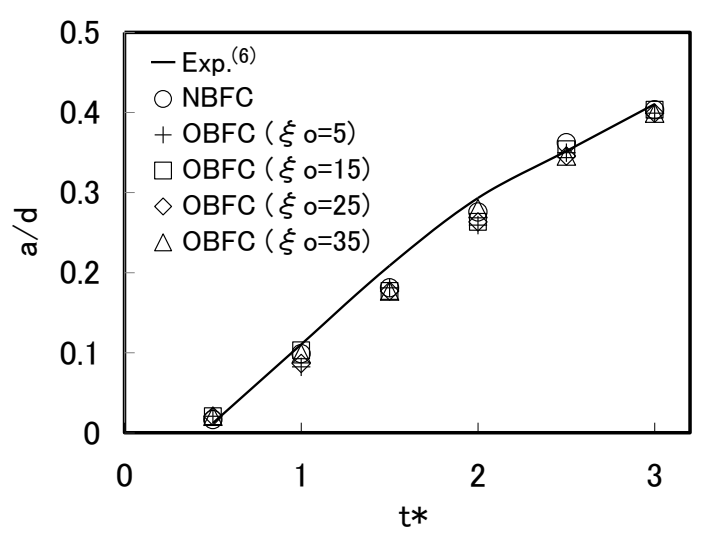

(b) $x$-coordinates of the main eddy core $a / d$

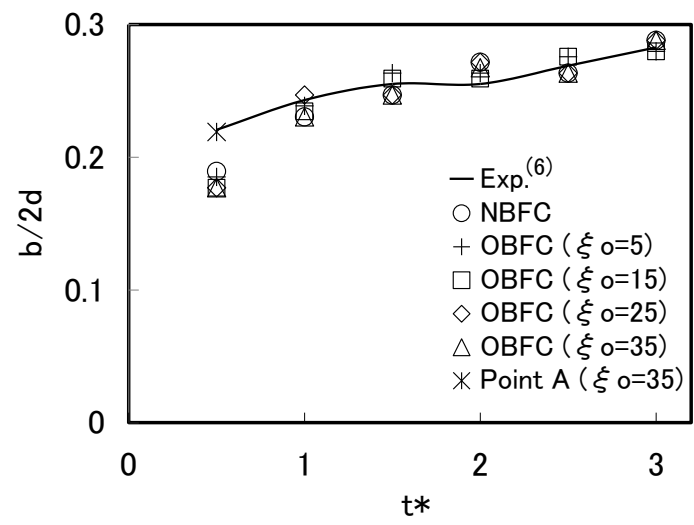

(c) $y$-coordinates of the main eddy core $b / 2 d$

Fig. 6. Comparison with the characteristics of the cylinder wake calculated by the orthogonal and non-orthogonal grids $(R e=550)$

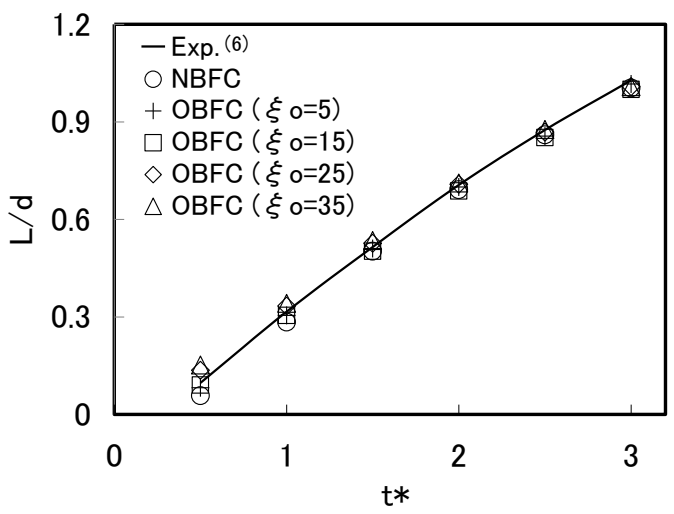

Fig. 7. Comparison with the closed cylinder wake length calculated by the orthogonal and non-orthogonal grids $(R e=31)$

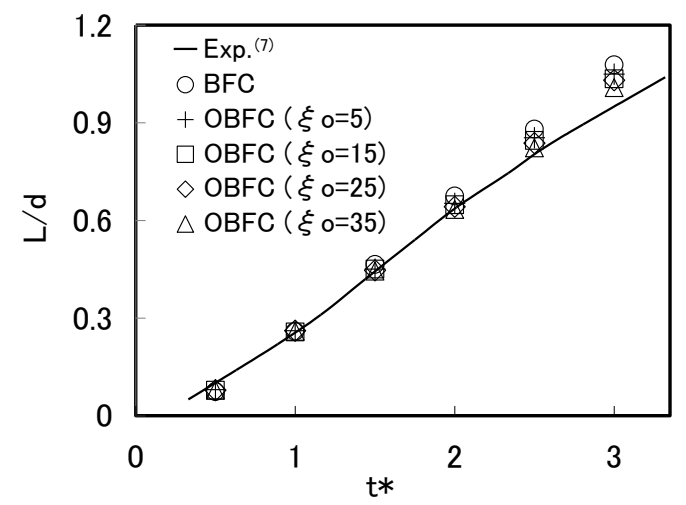

Fig. 8. Comparison with the closed cylinder wake length calculated by the orthogonal and non-orthogonal grids $(R e=1200)$
Accordingly, with the aim of making comparisons for a larger $R e$ and improving the reliability by comparison with the other previous measurements, a similar assessment was performed using the results for $R e=1200$ of Nagata et al.[7], who carried out similar measurements to Bouard et al.[6]. The results of the closed wake length $L / d$ are shown in Fig. 8 . As can be understood from Fig. 8, the calculated results for $L / d$ are slightly larger than the measurement results by Nagata et al.[7] for a later non-dimensional time $t^{*}$. Then, also in this comparison with the results by Nagata et al.[7], the differences between the measured results and the calculated results are more marked on the narrower orthogonal grid. For $t^{*}=3.0$, the calculated result using the $\xi o=35$ OBFC grid agrees with the measured result within less than $5 \%$, but the calculated results using the NBFC grid differ from them by about $12 \%$. Therefore, in this case, as same as the case for $R e=550$, the difference is also large when $R e$ is large.

As mentioned above, the effectiveness of grid orthogonality is confirmed on the geometrical parameters of the closed wake. Then, by the same manner, the calculated wall pressure will be discussed in the below. Fig.9 shows this result, and the calculated wall pressure coefficient $C_{p}$ is compared with the analysis result of Collins and Dennis [8]. In this Fig. 9, the calculated results are heavily influenced by the grid orthogonality. The conformity between this calculation and Dennis's analysis has proportional relationship to the area of the orthogonal region. The result calculated by the NBFC grid has larger difference with the analysis of Dennis, and the result by the $\xi_{0}=35$ OBFC grid has smaller difference with it. In this calculation, $\psi-\omega$ method is used. Consequently, the orthogonality on the wall is very important for the boundary condition of $\omega$, and in this case, the orthogonality may influence the accuracy of the calculation much more than velocity - pressure method. However, the effectiveness of grid orthogonality is obvious.

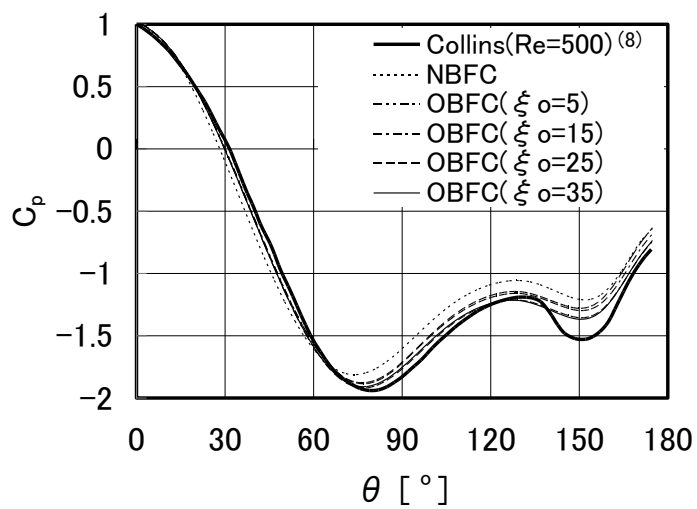

Fig. 9. Comparison with the wall pressure coefficient calculated by the orthogonal and non-orthogonal grids $\left(R e=550, t^{*}=1.4\right)$

\section{CONCLUSION}

In this study, without changing the coordinate lines along the flow direction and the boundary point positions, coordinate lines are newly generated to be orthogonal to the coordinate lines in the flow direction. Then, by this means, the effectiveness of the orthogonal grid could be investigated. The results indicate that the calculation results agree better 
with the visualization and the analysis results for a wider orthogonal region in the coordinate system. In other words, we verified that the orthogonal grid is important in the numerical calculation and the proposed straightforward orthogonal grid generation method is sufficiently effective. Additionally, the effect of this orthogonal grid is greater when $R e$ is larger, and in the case when $R e$ is small, the merits of the orthogonal grid are not readily reflected in the calculated results. However, with the actual phenomenon, since $R e$ is very big, we consider that the orthogonality of the numerical calculation grid is very important.

\section{REFERENCES}

[1] J. F. Thompson, F. C. Thames, and C. W. Mastin, "TOMCAT - A Code for Numerical Generation of Boundary Fitted Curvilinear Coordinate Systems on Fields Containing Any Number of Arbitrary Two-Dimensional Bodies," Journal of Computational Physics, vol. 24 pp. 274-302, 1977.

[2] J. F. Thompson, Z. U. A Warsi, and C. W. Mastin, Numerical Grid Generation-Foundations and Applications, Elsevier Science Publishing Co. Inc., ch. 1, pp. 2, 1985.

[3] K. Sugizaki and S. Kawata, "Three-dimensional grid generation," Software Science, vol. 97, no. 391, pp. 9-16, Nov. 1997.

[4] V. Akcelik, B. Jaramaz, and O. Ghattas, "Nearly Orthogonal Two-Dimensional Grid Generation with Aspect Ratio Control," Journal of Computational Physics, vol. 171, pp. 805-821, 2001.
[5] F. C. Thames, J. F. Thompson, and C. W. Mastin, "Numerical Solution of the Navier-Stokes Equations for Arbitrary Two-Dimensional Airfoils," in Proc. NASA Conf. Aerodynamic Analyses Requiring Advanced Computers, NASA SP-347, pp. 469-530, 1975.

[6] R. Bouard and M. Coutanceau, "The Early Stage of Development of the Wake Behind an Impulsively Started Cylinder for $40<R e<10^{4}$,' Journal of Fluid Mechanics, vol. 101, no. 3, pp. 583-607, 1980.

[7] H. Nagata, H. Funada, and T. Matui, "Unsteady Flows in the Vortex Region behind a Circular Cylinder Started Impulsively: 2nd Report, Velocity Field and Circulation," Transactions of the Japan Society of Mechanical Engineers Edition B, vol. 51, no. 463, pp. 748-755, 1985.

[8] W. M. Collins and S. C. R. Dennis, "Flow past an impulsively started circular cylinder," Journal of Fluid Mechanics, vol. 60, no. 1, pp. 105-127, 1973.

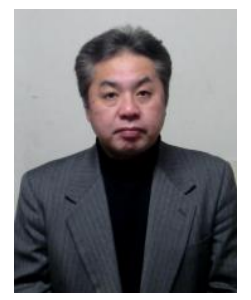

Ichiro Nakane received B. Eng. in 1983, M.Eng. in 1985 and PhD. in 2004 from Keio University. He has published and presented 67 papers in International \& National Conferences and Journals. Currently he is working as Associate Professor of the Mechanical Engineering Department, Kanagawa Institute of Technology, Atsugi, Kanagawa, Japan. His research interest areas are the following; Turbulent Flow, Swirling Flow, Two Phase Flow, Turbulent Transition, CFD, Numerical Simulation, Traffic Flow, Air Pollution, Wing in Ground Effect Vehicle, Wireless Electricity Transmission. 